\title{
Hyperbolic Moment Equations Using Quadrature-Based Projection Methods
}

\author{
J. Koellermeier and M. Torrilhon \\ Department of Mathematics, RWTH Aachen University, Aachen, Germany
}

\begin{abstract}
Kinetic equations like the Boltzmann equation are the basis for various applications involving rarefied gases. An important problem of many approaches since the first developments by Grad is the desired global hyperbolicity of the emerging set of partial differential equations. Due to lack of hyperbolicity of Grad's model equations, numerical computations can break down or yield nonphysical solutions. New hyperbolic PDE systems for the solution of the Boltzmann equation can be derived using quadrature-based projection methods. The method is based on a non-linear transformation of the velocity to obtain a Lagrangian velocity phase space description in order to allow for physical adaptivity, followed by a series expansion of the unknown distribution function in different basis functions and the application of quadrature-based projection methods.In this paper, we extend the proof for global hyperbolicity of the quadrature-based moment system system to arbitrary dimensions, utilizing quadrature-based projection methods for tensor product Hermite basis functions. The analytical computation of the eigenvalues shows the proposed correspondence to the Hermite quadrature points.
\end{abstract}

Keywords: kinetic equation, hyperbolicity, quadrature

PACS: 02.30.Jr, 05.20.Dd

\section{INTRODUCTION}

The solution of kinetic equations like the Boltzmann Equations requires an efficient and yet robust discretization of the velocity space to allow for both physical adaptivity and stability of the numerical method. The standard method to discretize the resulting equation in velocity space has been developed by Grad in [1] and relies on the expansion of the distribution function in Hermite polynomials. The drawback of this method is that the resulting PDE system can loose hyperbolicity for certain values of higher moments, see e.g. [2]. In these cases, numerical methods can become unstable because the problem is ill-posed.

Existing hyperbolic models are for example given in [3] where multi-variate Pearson-IV-Distributions are used as an ansatz or in [4] where a maximum entropy approach ensures the hyperbolicity.

Another method by Cai et al. relies on a truncation of the expansion at certain steps of the derivation and has been successfully applied to different problems (see [5]). This work has recently been supplemented with a solid theoretical basis in [6], where the procedure is generalized.

The novel quadrature-based projection methods have been first introduced in [7]. This approach computes the integrals during the projection by Gaussian quadrature which modifies the emerging system and guarantees hyperbolicity for a wide range of cases, provided some simple conditions are fulfilled. Up to now there is only a proof of hyperbolicity in the one-dimensional case using Hermite polynomials as ansatz functions, but the framework is able to handle multi-dimensional settings as well.

The main part of this paper is concerned with the extension of the proof given in [8] to arbitrary dimensions and more flexible basis expansions. The developed framework can be applied to this setting analogously to the one-dimensional case as we will show. This results in a straightforward proof and makes it possible to calculate the eigenvalues of the system analytically.

The paper is organized as follows: We first explain the basic concepts and recall the equations derived in [8] using quadrature-based projection methods, along with the conditions for hyperbolicity of the emerging system. A proof for global hyperbolicity of the PDE system in the general $d$-dimensional case is detailed in the main section. The paper ends with a short conclusion.

Proceedings of the 29th International Symposium on Rarefied Gas Dynamics

AIP Conf. Proc. 1628, 626-633 (2014); doi: 10.1063/1.4902651

(C) 2014 AIP Publishing LLC 978-0-7354-1265-1/\$30.00 


\section{TRANSFORMED BOLTZMANN TRANSPORT EQUATION}

We consider the Boltzmann equation without external force for the evolution of the mass density function $f(t, \underline{x}, \underline{c})$

$$
\frac{\partial}{\partial t} f(t, \underline{x}, \underline{c})+c_{i} \frac{\partial}{\partial x_{i}} f(t, \underline{x}, \underline{c})=S(f)
$$

where we assume a $d$-dimensional setting, i.e. $\underline{x} \in \mathbb{R}^{d}$ and velocity $\underline{v} \in \mathbb{R}^{d}$. Index notation is used whenever the indices are no further specified, e.g. in the transport term of Equation (1). The collision operator $S(f)$ on the right-hand side will be neglected throughout this paper as we focus on the transport part of Equation (1). Models for the right-hand side can be found in [9], [10] and [11].

The macroscopic quantities density $\rho(t, \underline{x})$, velocity $\underline{v}(t, \underline{x})$ and energy $\theta(t, \underline{x})$ of the distribution function can be computed by integration over the velocity space (we will omit the arguments most of the time for better readability).

$$
\rho=\int_{\mathbb{R}^{d}} f(t, \underline{x}, \underline{c}) d \underline{c}, \quad \rho \underline{v}=\int_{\mathbb{R}^{d}} \underline{c} f(t, \underline{x}, \underline{c}) d \underline{c}, \quad d \cdot \rho \theta=\int_{\mathbb{R}^{d}}|\underline{c}-\underline{v}|^{2} f(t, \underline{x}, \underline{c}) d \underline{c} .
$$

Multiplication of Eq.(1) with $\left(1, \underline{c}, c_{i}^{2}\right)$ followed by integration over the velocity space yields the conservation laws of mass, momentum and energy, see e.g. [12].

We recall the transformed version of the Boltzmann Eq. (1) that essentially uses a Lagrangian velocity phase space and thus exhibits physical adaptivity which allows for efficient and yet simple discretizations, introduced also in [13].

As described in [7], the velocity is transformed in a highly non-linear way to allow for intrinsic physical adaptivity of the scheme. We shift the microscopic velocity $\underline{c}$ by its macroscopic counterpart $\underline{v}$ and scale by the standard deviation $\sqrt{\theta}$. The corresponding Galilei-invariant variable transformation reads

$$
\underline{c} \mapsto \frac{\underline{c}-\underline{v}(t, \underline{x})}{\sqrt{\theta(t, \underline{x})}}=: \underline{\xi}(t, \underline{x}, \underline{c}) .
$$

Every Maxwellian in $\underline{c}$-space is thus transformed to a Gaussian in $\underline{\xi}$-space scaled with density $\rho$, as depicted in [7]. We furthermore use a scaled distribution function $\widetilde{f}(t, \underline{x}, \underline{\xi})$, defined by

$$
f(t, \underline{x}, \underline{\xi})=\frac{\rho}{\sqrt{\theta}^{d}} \tilde{f}(t, \underline{x}, \underline{\xi}) .
$$

According to [7] and with the help of the convective time derivative $D_{t}:=\partial_{t}+v_{i} \partial_{x_{i}}$, Equation (1) transforms to

$$
\begin{array}{ll}
\left(\frac{1}{\rho} D_{t} \rho-\frac{d}{2 \theta} D_{t} \theta\right) \widetilde{f}+D_{t} \widetilde{f}+\sqrt{\theta} \xi_{j}\left(\left(\frac{1}{\rho} \partial_{x_{j}} \rho-\frac{d}{2 \theta} \partial_{x_{j}} \theta\right) \widetilde{f}+\partial_{x_{j}} \tilde{f}\right)+ & \\
\partial_{\xi_{j}} \widetilde{f}\left(-\frac{1}{\sqrt{\theta}}\left(D_{t} v_{j}+\sqrt{\theta} \xi_{i} \partial_{x_{i}} v_{j}\right)-\frac{1}{2 \theta} \xi_{j}\left(D_{t} \theta+\sqrt{\theta} \xi_{i} \partial_{x_{i}} \theta\right)\right) & =0 .
\end{array}
$$

This PDE for the vector of unknowns $\underline{u}=\left(\rho, v_{1}, \ldots, v_{d}, \theta, \tilde{f}\right)^{T}$ can be written in the following compact notation

$$
\underline{\underline{\Lambda}} \underline{A} D_{t} \underline{u}+\underline{\underline{\Lambda}} \sqrt{\theta} \xi_{i} \underline{A} \partial_{x_{i}} \underline{u}=0,
$$

using the definitions

$$
\begin{gathered}
\underline{A}=\left(\tilde{f},-\frac{\partial \tilde{f}}{\partial \xi_{1}}, \ldots,-\frac{\partial \tilde{f}}{\partial \xi_{d}},-d \tilde{f}-\xi_{j} \frac{\partial \tilde{f}}{\partial \xi_{j}}, 1\right) \in \mathbb{R}^{d+3}, \\
\underline{\underline{\Lambda}}=\left(\begin{array}{ccc}
\frac{1}{\rho} & \frac{1}{\sqrt{\theta}} \underline{\underline{I_{d}}} & \\
& \frac{1}{2 \sqrt{\theta}} & \\
& & 1
\end{array}\right) \in \mathbb{R}^{(d+3) \times(d+3)} .
\end{gathered}
$$


Equation (6) is underdetermined, as $\rho, \underline{v}$ and $\theta$ are not known. But the definition of the macroscopic variables according to Eq. (2) leads to $d+2$ additional algebraic equations that formally close the system.

It was shown in [8] that point evaluations of Eq. (6) together with a reasonable finite difference approximation of the derivatives $\partial_{\xi_{j}} \widetilde{f}$ do in general not lead to a globally hyperbolic system as eigenvalues of the system matrix depend on the unknowns. A deficiency that is removed with the help of quadrature-based projection methods, as explained in the following Section.

\section{GENERAL FRAMEWORK FOR HYPERBOLIC APPROXIMATION OF KINETIC EQUATIONS}

We expand the unknown distribution function $f(t, \underline{x}, \underline{\xi})$ around its equilibrium Maxwellian with respect to basis functions $\phi_{\alpha}(\underline{\xi})$ and corresponding coefficients $\kappa_{\alpha}(t, \underline{x})$, obtaining

$$
\widetilde{f}(t, \underline{x}, \underline{\xi})=\sum_{\alpha=1}^{n} \kappa_{\alpha}(t, \underline{x}) \phi_{\alpha}(\underline{\xi}) .
$$

with differentiable ansatz functions $\phi_{\alpha}(\underline{\xi})$.

After insertion of Ansatz (9) into Eq. (6), we multiply with test functions $\psi_{\beta}(\underline{\xi})$ for $\beta=1, \ldots, n$ and integrate over the velocity space to obtain a system of equations for the unknowns $\rho, \underline{v}, \theta$ and the basis coefficients $\kappa_{\alpha}$. This can be interpreted as a projection with projection operator $P$

$$
P_{\beta}(g):=\left\langle g, \psi_{\beta}\right\rangle=\int_{\mathbb{R}^{d}} g \psi_{\beta} \underline{d \underline{\xi}} .
$$

As the definition of $P$ using exact integration does not lead to global hyperbolic systems (see [5]), we use an approximative projection operator $Q$, that computes the integrals by Gauss-quadrature

$$
P_{\beta}(g) \approx Q_{\beta}(g):=\sum_{k=1}^{n} w_{k} g\left(\underline{\xi_{k}}\right) \psi_{\beta}\left(\underline{\xi_{k}}\right) \text {. }
$$

with positive quadrature weights $w_{k}$ and pairwise distinct quadrature points $\xi_{k} \in \mathbb{R}^{d}$, for $k=1, \ldots, n$.

Applying $Q_{\beta}$ for $\beta=1, \ldots, n$ to Eq. (6) with Expansion (9) inserted, the emerging PDE system can be written in the following form

$$
\underline{\underline{\Psi}}^{T} \underline{\underline{W A \Lambda}} \frac{D}{D t} \underline{\widetilde{u}}+\sum_{i=1}^{d} \underline{\underline{\Psi}}^{T} \underline{\underline{W}} \underline{\underline{\Xi}} \underline{\underline{A \Lambda}} \sqrt{\theta} \frac{\partial}{\partial x_{i}} \underline{\widetilde{u}}=0
$$

with unknowns

$$
\underline{\widetilde{u}}=\left(\rho, v_{1}, \ldots, v_{d}, \theta, \kappa_{0}, \ldots, \kappa_{n}\right)^{T} \in \mathbb{R}^{n+d+2},
$$

a columnwise defined system matrix

$$
\underline{\underline{A}}=\left(\underline{\underline{\Phi}},-\frac{\partial \underline{\underline{\Phi}}}{\partial \xi_{1}} \underline{\kappa}, \ldots,-\frac{\partial \underline{\underline{\Phi}}}{\partial \xi_{d}} \underline{\kappa},-d \underline{\underline{\Phi}}-\underline{\underline{\Xi}} \underline{\underline{\Xi_{j}}} \frac{\partial \underline{\underline{\Phi}}}{\partial \xi_{j}} \underline{\underline{\Phi}}, \underline{\underline{\Phi}}\right) \in \mathbb{R}^{n \times(n+d+2)}
$$

consisting of matrices (for $i, j=1, \ldots, n$ and $k=1, \ldots, d$ )

$$
\begin{gathered}
\underline{\underline{\Psi}}_{i, j}=\psi_{j}\left(\underline{\xi_{i}}\right), \quad \underline{\Phi}_{i, j}=\phi_{j}\left(\underline{\xi_{i}}\right), \quad\left(\frac{\partial \underline{\underline{\Phi}}}{\partial \xi_{k}}\right)_{i, j}=\left.\partial_{\xi_{k}} \phi_{j}\right|_{\underline{\xi_{i}}} \\
\left(\underline{\underline{\Xi_{k}}}\right)_{i, j}=\left(\underline{\xi}_{i}\right)_{k} \delta_{i j}, \quad \underline{\underline{W}}_{i, j}=\omega_{i} \delta_{i j}
\end{gathered}
$$

as well as $\underline{\underline{\Lambda}}$ from Eq. (26) with $\underline{I_{n}}$ instead of 1 as last entry.

It is already clear, that System (12) is not closed, as its $n$ equations include $n+d+2$ unknowns. A model reduction is explained in the following according to the procedure described in [8]. 

(2)

Inserting the scaled distribution function from Eq. (4) and using (9), we get $d+2$ compatibility conditions from Eqs.

$$
1=\kappa_{\alpha}\left\langle\phi_{\alpha}(\underline{\xi}), 1\right\rangle, \quad 0=\kappa_{\alpha}\left\langle\phi_{\alpha}(\underline{\xi}), \xi_{i}\right\rangle, \text { for } i=1, \ldots, d, \quad d=\kappa_{\alpha}\left\langle\phi_{\alpha}(\underline{\xi}), \xi_{i}^{2}\right\rangle
$$

The compatibility conditions (17) can be used to reduce the number of unknowns in the PDE system to obtain a closed PDE system similar to the general procedure described in [14]. We thus use $\underline{\underline{Q}} \in \mathbb{R}^{l \times n}, \underline{c}=(1,0, \ldots, 0, d)^{T} \in \mathbb{R}^{l}$ and write the $l=d+2$ Eqs. (17) in matrix-vector form to define $\underline{\underline{Q}}$ by

$$
\underline{\underline{Q}} \underline{\kappa}=\underline{c} .
$$

Now we decompose $\underline{\underline{Q}} \in \mathbb{R}^{l \times n}$ as follows (e.g. using singular value decomposition):

$$
\underline{\underline{Q}}=\underline{\underline{S M T^{-1}}} \text {, with regular } \underline{\underline{S}} \in \mathbb{R}^{l \times l}, \underline{\underline{M}}=(\underline{\underline{\underline{Q}}}, \underline{\underline{0}}) \in \mathbb{R}^{l \times n} \text {, regular } \underline{\underline{\underline{Q}}} \in \mathbb{R}^{l \times l} \text { and regular } \underline{\underline{T}} \in \mathbb{R}^{n \times n} \text {. }
$$

We can then solve for the first $l$ variables of a transformed set of variables $\underline{\beta} \in \mathbb{R}^{n}$ using

$$
\underline{\widehat{\beta}}=\left(\frac{\beta_{0}}{\underline{\beta}}\right):=\underline{\underline{T}}^{-1} \underline{\kappa} \Rightarrow \underline{\beta}_{0}=\underline{\underline{Q}}^{-1} \underline{\underline{S}}^{-1} \underline{c}
$$

and then close the system of PDEs by inserting

$$
\underline{\kappa}=\underline{\underline{T}}\left(\frac{\beta_{0}}{\underline{\beta}}\right)=\left(\underline{\underline{T_{1}}}, \underline{\underline{T_{2}}}\right)\left(\frac{\beta_{0}}{\underline{\beta}}\right)=\underline{\underline{T_{1}}} \underline{\underline{Q}}^{-1} \underline{\underline{S}}^{-1} \underline{\underline{c}}+\underline{\underline{T_{2}}} \underline{\beta},
$$

with a proper splitting of the columns of $\underline{\underline{T}}=\left(\underline{\underline{T_{1}}}, \underline{\underline{T_{2}}}\right)$.

With the help of the compatibility conditions, the set of $n$ basis coefficients $\underline{\kappa}$ is thus reduced to a set of $n-l$ variables $\beta$ which leads to a closed PDE system with the same number of equations and unknowns.

Rewriting System (12) with the help of the reduced variables, we get

$$
\underline{\underline{\Psi}}^{T} \underline{\underline{W}} \underline{\underline{A_{\beta}}} \underline{\underline{\underline{\Lambda}}} \beta \frac{D}{D t} \underline{u}+\sum_{i=1}^{d} \underline{\underline{\Psi}}^{T} \underline{\underline{W}} \underline{\underline{\underline{\Xi_{i}}}} \underline{\underline{A_{\beta}}} \underline{\underline{\underline{\Lambda}}} \underline{\underline{\beta}} \sqrt{\theta} \frac{\partial}{\partial x_{i}} \underline{u}=0
$$

with a columnwise defined system matrix $\underline{\underline{A_{\beta}}}$ of which the first $d+2$ columns include the transformed coefficients $\underline{\beta}$ :

$$
\underline{\underline{A_{\beta}}}=\left(\underline{\underline{\Phi}}_{\underline{\beta}},-\frac{\partial \underline{\underline{\Phi}}}{\partial \xi_{1}}, \ldots,-\frac{\partial \underline{\underline{\Phi}}}{\partial \xi_{d}},-d \underline{\underline{\Phi}}_{\underline{\beta}}-\underline{\underline{\Xi}}_{j} \frac{\partial \underline{\underline{\Phi}}}{\partial \xi_{j}}, \underline{\underline{\Phi}} 2\right) \in \mathbb{R}^{n \times n} .
$$

Additionally, we use the definition of the matrices (for $i, j=1, \ldots, n$ and $k=1, \ldots, d$ )

$$
\begin{aligned}
& \underline{\underline{\Phi}}_{\underline{\beta}}=\underline{\underline{\Phi}}\left(\underline{\underline{T_{1}}} \underline{\underline{Q}}^{-1} \underline{\underline{S}}^{-1} \underline{c}+\underline{\underline{T_{2}}} \underline{\beta}\right), \\
& \frac{\partial \underline{\underline{\Phi}}}{\partial \bar{\xi}_{k} \underline{\beta}}=\frac{\partial \underline{\underline{\Phi}}}{\partial \xi_{k}}\left(\underline{\underline{T}_{1}} \underline{\underline{Q}}^{-1} \underline{\underline{S}}^{-1} \underline{\underline{c}}+\underline{\underline{T_{2}}} \underline{\beta}\right), \\
& \underline{\underline{\Lambda}}_{\underline{\beta}}=\left(\begin{array}{cccc}
\frac{1}{\rho} & & & \\
& \frac{1}{\sqrt{\theta}} \underline{\underline{I_{d}}} & & \\
& & \frac{1}{2 \sqrt{\theta}} & \\
& & & \underline{\underline{I_{n-d-2}}}
\end{array}\right) \in \mathbb{R}^{n \times n} .
\end{aligned}
$$

The subscript $\beta$ thus denotes a dependence of the corresponding matrix on the unknowns $\beta$.

The important question of hyperbolicity of System (22) is addressed by Theorem 1 in [8] and requires essentially only the regularity of matrices $\underline{\underline{\Psi}}, \underline{\underline{W}}, \underline{\underline{\Lambda}} \underline{\underline{\underline{\Lambda}}}$ and $\underline{\underline{A_{\beta}}}$, of which the regularity of matrix $\underline{\underline{A_{\beta}}}$ is the only non-trivial task for most cases. 
Provided these requirements, the generalized eigenvalues $\lambda_{k}$ for $k=1, \ldots, n$ are all real and read

$$
\lambda_{k}=\sum_{i=1}^{d} n_{i}\left(\left(\underline{\xi_{k}}\right)_{i} \sqrt{\theta}+v_{i}\right), \text { for } k=1, \ldots, n .
$$

After the validation of the conditions for the one-dimensional case in previous papers, we will show global hyperbolicity in the general $d$-dimensional case in the following Section.

\section{GLOBALLY HYPERBOLIC SYSTEM USING HERMITE ANSATZ AND QUADRATURE IN D DIMENSIONS}

A proof of global hyperbolicity for the one-dimensional system with Hermite ansatz functions can be found in [8]. We will now extend the proof to the general case of $d$ dimensions in position and velocity space, i.e. $\underline{x}, \xi \in \mathbb{R}^{d}$.

We consider the following basis expansion for the scaled distribution function $f$ in orthonormal basis functions

$$
\widetilde{f}(t, x, \xi)=\sum_{\alpha_{i} \leq N_{i}} \kappa_{\underline{\alpha}}(t, \underline{x}) \phi_{\underline{\alpha}}(\underline{\xi}),
$$

for a $d$-dimensional multi-index $\underline{\alpha} \in \mathbb{N}^{d}$ and a vector $\underline{N}=\left(N_{0}, \ldots, N_{d}\right) \in \mathbb{N}^{d}$ specifying the moment theory. The total number of unknown basis coefficients is denoted as $N=\prod_{i=1}^{d}\left(N_{i}+1\right)$.

The corresponding basis functions are the tensor product of one-dimensional Hermite functions

$$
\phi_{\underline{\alpha}}(\underline{\xi})=\prod_{i=1}^{d} \phi_{\alpha_{i}}\left(\xi_{i}\right), \quad \phi_{i}(x)=H e_{i}(x) \frac{1}{\sqrt{2 \pi}} e^{-x^{2} / 2} \quad \text { and test functions } \psi_{\underline{\alpha}}(\underline{\xi})=\prod_{i=1}^{d} H e_{i}\left(\xi_{i}\right),
$$

with normalized Hermite polynomial $H e_{i}$ of degree $i$ such that the following recursion formulas hold

$$
\frac{\partial \Phi_{\underline{\alpha}}}{\partial \xi_{i}}=-\sqrt{\alpha_{i}+1} \Phi_{\underline{\alpha}+\underline{e_{i}}}, \quad \xi_{i} \frac{\partial \Phi_{\underline{\alpha}}}{\partial \xi_{i}}=-\sqrt{\alpha_{i}+1}\left(\sqrt{\alpha_{i}+2} \Phi_{\underline{\alpha}+2 \underline{e_{i}}}+\sqrt{\alpha_{i}+1} \Phi_{\underline{\alpha}}\right) .
$$

Note that this ansatz (28) and (29) exhibits some flexibility, because different choices for $N_{i}$ can be chosen to capture the different behaviour of the distribution function in each spatial direction. We will nevertheless loose rotational invariance with this separation ansatz.

The quadrature projection is done using multi-dimensional Gauss-Hermite quadrature as follows

$$
\int_{\mathbb{R}^{d}} g(\underline{\xi}) d \underline{\xi} \approx \sum_{i_{1}=0}^{N_{1}} \ldots \sum_{i_{d}=0}^{N_{d}} g\left(\left(\xi_{1}\right)_{i_{1}}, \ldots,\left(\xi_{d}\right)_{i_{d}}\right) w_{1, i_{1}} \cdot \ldots w_{d, i_{d}},
$$

using quadrature points $\left(\xi_{i}\right)_{j}$ and weights $w_{i, j}$ given by

$$
\begin{aligned}
\left(\xi_{i}\right)_{j} & =j \text {-th root of } H e_{N_{i}+1}\left(\xi_{i}\right), \text { for } i=1, \ldots, d, j=0, \ldots, N_{i} \\
w_{i, j} & =j \text {-th weight of quadrature formula in direction } i, \text { for } i=1, \ldots, d, j=0, \ldots, N_{i}
\end{aligned}
$$

A compact notation for the quadrature formula is

$$
\int_{\mathbb{R}^{d}} g(\underline{\xi}) d \underline{\xi} \approx \sum_{k=1}^{N} g\left(\underline{\xi_{k}}\right) w_{k},
$$

with the total number of quadrature points equal to the number of basis coefficients $N$ and a consecutive ordering of the quadrature points according to

$$
\begin{aligned}
\underline{\xi_{k}} & \in\left\{\left(\xi_{i_{1}}, \ldots, \xi_{i_{d}}\right) \mid i_{j} \in\left\{0, \ldots, N_{j}\right\}, j=1, \ldots, d\right\} \\
w_{k} & \in\left\{\Pi_{j=1}^{d} w_{j, i_{j}} \mid i_{j} \in\left\{0, \ldots, N_{j}\right\}, j=1, \ldots, d\right\}
\end{aligned}
$$

The setting described above enables us to prove the following central theorem of this paper:

Theorem 1 Using tensor product Hermite basis and test functions as specified in Eqs. (29) together with GaussHermite quadrature, the conditions of Theorem 1 in [8] are fulfilled and the resulting PDE system is globally hyperbolic. 
Proof We first reduce the number of unknowns as described in using the decomposition (19) and state the compatibility conditions derived using Equation (17) as

$$
1=\kappa_{\underline{0}}, \quad 0=\kappa_{\underline{e_{i}}}, \text { for } i=1, \ldots, d, \quad d=d \kappa_{\underline{0}}+\sqrt{2} \sum_{i=1}^{d} \kappa_{2 \underline{e_{i}}} .
$$

We order the basis coefficients introducing the index set $N_{\mathcal{K}}=\left\{\underline{\alpha} \in \mathbb{N}^{d}|1<| \underline{\alpha} \mid, \alpha_{i} \leq N_{i}, \underline{\alpha} \neq 2 \underline{e_{i}}, i=1, \ldots, d\right\}$

$$
\kappa=\left(\kappa_{\underline{0}}, \kappa_{\underline{e_{i}}}, \kappa_{2 \underline{e_{i}}}, \kappa_{\underline{\alpha} \in \bar{N}_{\kappa}}\right) .
$$

to write Equations (37) in matrix vector form as $\underline{\underline{Q}} \underline{\underline{\kappa}}=\underline{c}$, see (18), with

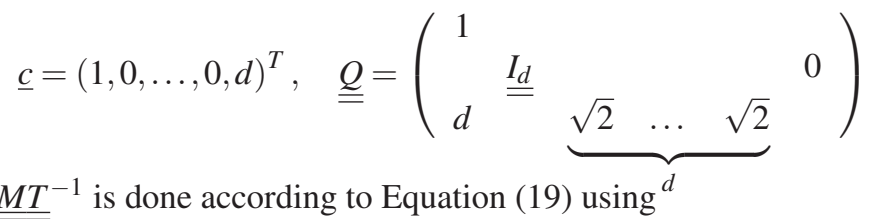

A decomposition $\underline{\underline{Q}}=\underline{\underline{S M T}}{ }^{-1}$ is done according to Equation (19) using ${ }^{d}$

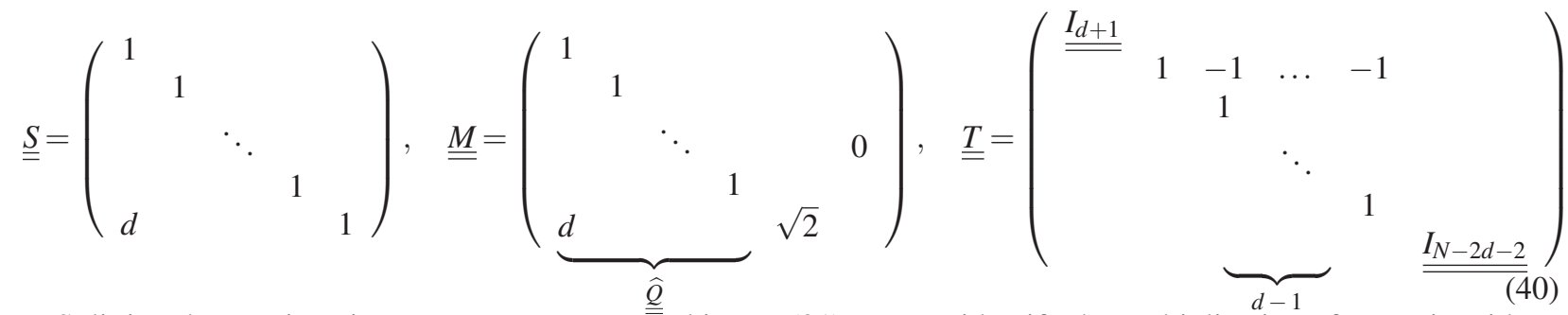

Splitting the matrix $\underline{\underline{T}}$ into two parts as proposed in Eq. (21), we can identify the multiplication of a matrix with $\underline{\underline{T_{2}}}$ from the right as deletion of the first $d+1$ columns and subtraction of the $d+2^{\text {nd }}$ column from columns $d+3$ up to $2 d+2$. Multiplication with $\underline{T}_{1}$ extracts the first $d+2$ columns.

The unknown vector $\underline{\underline{\kappa}}$ can be rewritten with only $N-d-2$ variables as

$$
\underline{\underline{\kappa}}=\left(1,0, \ldots, 0,-\sum_{i=1}^{d} \kappa_{2 \underline{e_{i}}}, \underset{\underline{\alpha} \in N_{\kappa}}{\kappa_{\alpha}}\right)^{T},
$$

which corresponds to the solution of Eqs. (37) for the first $d+2$ unknowns.

We will now derive matrix $A_{\beta}$ in (23) columnwise in order to show regularity of $\underline{\underline{A_{\beta}}}$ later.

For better readability, we introduce a set of multi-indices $N_{\beta_{0}}=\left\{\underline{\underline{0}}, \underline{e_{1}}, \ldots, \underline{e_{d}}, \overline{\overline{2 e_{1}}}\right\}$, such that the corresponding basis coefficients $\kappa_{\underline{\alpha}}$ for $\underline{\alpha} \in N_{\beta_{0}}$ are all the coefficients $\underline{\beta_{0}}$, that we have explicitly calculated using the compatibility conditions, i.e. the first $d+2$ entries in $\underline{\kappa}$ and the remaining multi-indices form the set $N_{\beta}=\left\{\underline{\alpha} \in \mathbb{N}^{d}, \alpha_{i} \leq N_{i}, 1<\right.$ $\left.|\underline{\alpha}|, \underline{\alpha} \neq 2 \underline{e_{1}}\right\}$.

The last $\bar{N}-d-2$ columns of $\underline{A_{\beta}}$ (see (23) for the definition), identified by the corresponding multi-index $\underline{\alpha} \in N_{\beta}$, are $\underline{\underline{\Phi}} \underline{\underline{T_{2}}}$ and read

$$
\underline{\underline{\Phi}} \underline{\underline{T_{2}}} \underline{\underline{\alpha}}=\left(\underline{\Phi}_{\underline{\alpha}}-\underline{\Phi}_{2 \underline{e}_{1}} \sum_{j=2}^{d} \delta_{\underline{\alpha}, 2 e_{j}}\right), \text { for } \underline{\alpha} \in N_{\beta} .
$$

The first column $\underline{\underline{\Phi}}_{\underline{\beta}}$ can be written as

$$
\underline{\underline{\Phi}}_{\underline{\beta}}=\underline{\underline{\Phi}}_{\underline{0}}+\sum_{j=2}^{d}\left(\underline{\underline{\Phi}}_{2 \underline{e_{j}}}-\underline{\underline{\Phi}}_{2 \underline{e_{1}}}\right) \kappa_{2 \underline{e}_{j}}+\sum_{\underline{\alpha} \in N_{\kappa}} \underline{\underline{\Phi}}_{\underline{\alpha}} \kappa_{\underline{\alpha}}
$$


Columns 2 to $d+1$ can be simplified using the recursion formulas in (30)

$$
-\frac{\partial \underline{\underline{\Phi}}}{\partial \underline{\xi}_{i} \underline{\beta}}=\underline{\Phi}_{\underline{e_{i}}}-\sqrt{2} \underline{\underline{\Phi}}_{2 \underline{e_{1}}+\underline{e_{i}}} \sum_{j=2}^{d} \kappa_{2 \underline{e_{j}}}+\sum_{\underline{\alpha} \in N_{\beta}, \alpha_{i}<N_{i}} \underline{\underline{\alpha}}_{\underline{\alpha}+\underline{e_{i}}} \sqrt{\alpha_{i}+1} \kappa_{\underline{\alpha}}
$$

where the last sum only includes $\alpha_{i}<N_{i}$, because a value $\alpha_{i}=N_{i}$ would evaluate $\underline{\underline{\Phi}}_{\underline{\alpha}+e_{i}}$ to zero, as the $i$-th entry of each quadrature point is a root of the $N_{i}+1^{\text {st }}$ Hermite polynomial.

For the remaining $d+2^{\text {nd }}$ column, the term $\sum_{j=1}^{d} \xi_{j} \frac{\partial \Phi}{\partial \xi_{j} \beta}$ can be written as

$$
\sum_{j=1}^{d} \xi_{j} \frac{\partial \underline{\underline{\Phi}}}{\partial \xi_{j} \underline{\beta}}=-\sum_{j=1}^{d} \sum_{\alpha_{j}<N_{j}}\left(\alpha_{j}+1\right) \underline{\Phi}_{\underline{\alpha}} \kappa_{\underline{\alpha}}-\sum_{j=1}^{d} \sum_{\alpha_{j}+1<N_{j}} \sqrt{\alpha_{j}+1} \sqrt{\alpha_{j}+2} \underline{\Phi}_{\underline{\alpha}+2 \underline{e}_{j}} \kappa_{\underline{\alpha}},
$$

so that the entire column reads

$$
\begin{aligned}
-d \underline{\Phi}_{\underline{\beta}}-\sum_{j=1}^{d} \xi_{j} \frac{\partial \underline{\underline{\Phi}}}{\partial \xi_{j} \underline{\beta}} & =\sum_{j=1}^{d}\left(-\sum_{\alpha_{j}=N_{j}} \underline{\Phi}_{\underline{\alpha}} \kappa_{\underline{\alpha}}+\sum_{\alpha_{j}<N_{j}} \alpha_{j} \underline{\Phi}_{\underline{\alpha}} \kappa_{\underline{\alpha}}+\sum_{\alpha_{j}+1<N_{j}} \sqrt{\alpha_{j}+1} \sqrt{\alpha_{j}+2} \underline{\Phi}_{\underline{\alpha}+2 \underline{e}_{j}} \kappa_{\underline{\alpha}}\right) \\
& =\sqrt{2} \sum_{j=1}^{d} \underline{\Phi}_{2 e_{j}}+2 \sum_{j=2}^{d}\left(\underline{\Phi}_{2 e_{j}}-\underline{\Phi}_{2 \underline{e}_{1}}\right) \kappa_{2 e_{j}}+\sum_{\underline{\alpha} \in N_{\kappa}} \underline{\Phi}_{\underline{\alpha}} \underline{\gamma}_{\underline{\alpha}}
\end{aligned}
$$

for $\gamma_{\underline{\alpha}} \in \mathbb{R}$ including some coefficients $\kappa_{\underline{\alpha}}$ to sum up the higher order terms.

With the help of the expressions for the single columns, we can now easily go through the conditions of Theorem 1 in [8] and prove the central statement of this paper.

We have to check regularity of matrices $\underline{\underline{\Psi}}, \underline{\underline{W}}, \underline{\underline{\Lambda}}_{\beta}$ and $A_{\beta}$ in order to verify global hyperbolicity of System (22) in our setting.

$\underline{\Psi}$ is regular by construction, as its columns are point evaluations of each orthogonal test function.

$\underline{\bar{W}}$ is a regular diagonal matrix, because the quadrature weights are strict positive for multi-dimensional GaussHermite quadrature.

$\underline{\underline{\Lambda}}_{\beta}$ is regular, if and only if $\rho \neq 0$ and $\theta \neq 0$, this is true by assumption of Ansatz (4).

As derived above, every column of $A_{\beta}$ is a linear combination of some $\underline{\Phi}_{\alpha}$. Linear independence of its colums can be proved by checking where each column $\underline{\Phi}_{\underline{\alpha}}$ appears. In total, we must not have more than $N$ columns $\underline{\Phi}_{\alpha}$ involved and all columns of $A_{\beta}$ have to be linearly independent of each other.

(1) $\underline{\underline{\Phi}} \underline{\underline{T_{2}}}$ (see Eq. (42)) has linear independent columns $\left(\underline{\Phi}_{\underline{\alpha}}-\underline{\Phi}_{2 \underline{e}_{1}} \sum_{j=2}^{d} \delta_{\underline{\alpha}, 2 e_{j}}\right)$, for $\underline{\alpha} \in N_{\beta}$,

(2) $\underline{\Phi}_{\beta}$ (see Eq. (43)) is a linear combination of columns of $\underline{\underline{\Phi}} \underline{\underline{T_{2}}}$ plus an additional $\underline{\Phi}_{\underline{0}}$ and thus linearly independent of $\frac{\bar{\Phi}}{\partial \Phi} 2$

(3) $-\frac{\partial \Phi}{\partial \bar{\xi}_{i} \beta}$ (see Eq. (44)) is a linear combination of the columns of $\underline{\underline{\Phi}} \underline{\underline{T_{2}}}$ plus an additional $\underline{\Phi}_{\underline{e_{i}}}$ and thus linearly independent of the others

(4) $-d \underline{\underline{\Phi}}_{\underline{\beta}}-\sum_{j=1}^{d} \xi_{j} \frac{\partial \Phi}{\partial \xi_{j} \beta}$ (see Eq. (47)) is a linear combination of columns of $\underline{\underline{\Phi}} \underline{\underline{T_{2}}}$ plus an additional $\sqrt{2} \sum_{j=1}^{d} \underline{\Phi}_{2 e_{j}}$ and thus linearly independent of the others.

According to Eq.(27), the eigenvalues of the system matrix can be derived analytically and are closely connected with the Hermite roots. The following two-dimensional example in Fig.1 shows that the eigenvalues in fact lie on circles going through the origin and one of the quadrature points, which consists of Hermite roots.

\section{CONCLUSION}

After the presentation of the most important notations and previous results, we have described a very flexible ansatz using tensor product Hermite basis functions of different order in each spatial direction. This ansatz is capable of capturing the important features of the distribution function, e.g. for applications with certain pronounced directions. 

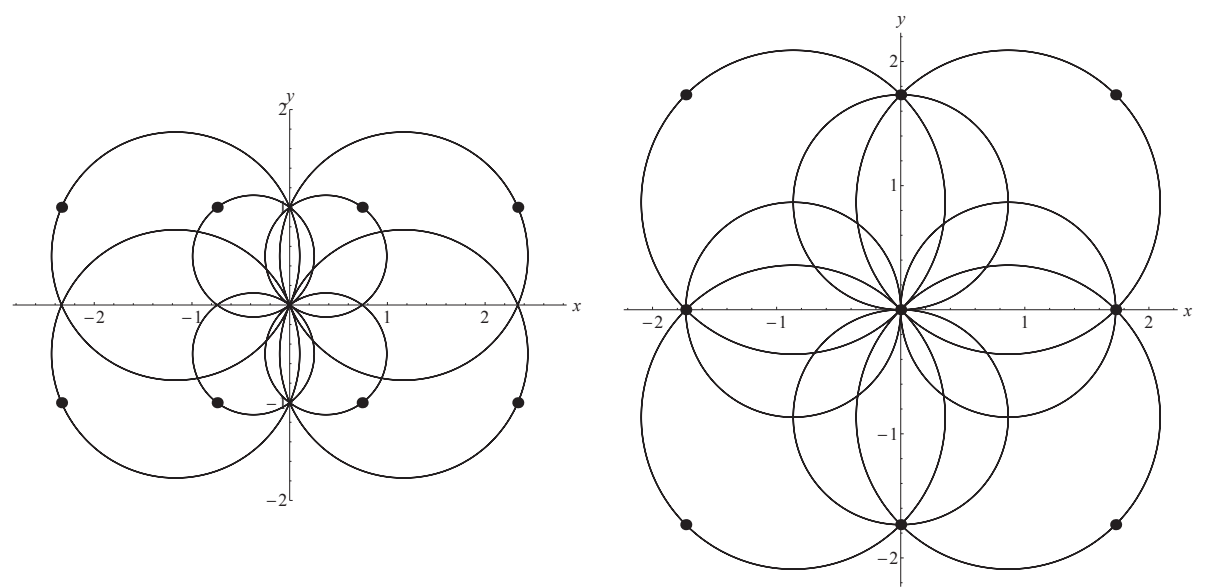

FIGURE 1. Eigenvalue plot of $2 \mathrm{D}$ example for asymmetric $N_{1}=3, N_{2}=1$ (left) and symmetric $N_{1}=N_{2}=2$ (right)

By extension of the proof for hyperbolicity from the one-dimensional case to the multi-dimensional case, we have shown another successful application of the general framework developed in [8]. With the help of simple quadrature rules and their properties, the necessary expressions could be derived and it was possible to show global hyperbolicity of the system of equations. Furthermore, the eigenvalues of the generalized system matrix could be computed analytically.

\section{ACKNOWLEDGMENTS}

This work has been carried out with the support of the German National Academic Foundation.

\section{REFERENCES}

1. H. Grad, Comm. Pure Appl. Math. 2, 331-407 (1949).

2. F. Brini, Continuum Mech. Thermodyn. 13, 1-8 (2001).

3. M. Torrilhon, Comm. Comput. Phys. 7, 639-673 (2010).

4. C. D. Levermore, Journal of Statistical Physics 83, 1021-1065 (1996).

5. Z. Cai, Y. Fan, and R. Li, Commun. Math. Sci. 11, 547-571 (2013).

6. Z. Cai, Y. Fan, and R. Li, "A Framework on Moment Model Reduction for Kinetic Equation," (submitted).

7. J. Koellermeier, Hyperbolic Approximation of Kinetic Equations Using Quadrature-Based Projection Methods, Master's thesis, RWTH Aachen University (2013).

8. J. Koellermeier, R. Schaerer, and M. Torrilhon, Kinet. Relat. Mod, 7, 531-549 (2014).

9. P. L. Bhatnagar, E. P. Gross, and M. Krook, Physical Review 94, 511-525 (1954).

10. C. Cercignani, The Boltzmann Equation and its Application, Springer, 1988.

11. S. Heinz, Statistical Mechanics of Turbulent Flows, Springer, 2003.

12. H. Struchtrup, Macroscopic Transport Equations for Rarefied Gas Flows, Springer, 2005.

13. P. Kauf, Multi-Scale Approximation Models for the Boltzmann Equation, Ph.D. thesis, ETH Zürich (2011).

14. E. Hairer, and G. Wanner, Solving Ordinary Differential Equations II, Stiff and Differential-Algebraic Problems, Springer Verlag, 2002. 